IRA-International Journal of Education \&

Multidisciplinary Studies

QUARTERLY

ISSN 2455-2526; Vol.16, Issue 04 (Oct.-Dec., 2020)

Pg. no. 217-223.

Institute of Research Advances

https://research-advances.org/index.php/IJEMS

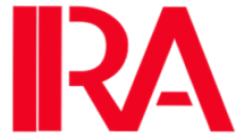

\title{
A Comparative Analysis of Verb Synonyms from the Perspective of Valency Structure in the Context of 'Provide and Offer'
}

\author{
Mei Hongxia \\ School of Foreign Languages, Yangtze University, China.
}

Type of Work: Peer-Reviewed

DOl: http://dx.doi.org/10.21013/jems.v16.n4.p2

How to cite this paper:
Hongxia, M. (2020). A Comparative Analysis of Verb Synonyms from the Perspective of Valency
Structure in the Context of 'Provide and Offer'. IRA International Journal of Education and
Multidisciplinary Studies (ISSN 2455-2526), 16(4), 217-223.
DOl: http://dx.doi.org/10.21013/jems.V16.n4.p2

(C) Institute of Research Advances.

This work is licensed under a Creative Commons Attribution-NonCommercial 4.0 International License subject to a proper citation to the publication source of the work.

Disclaimer: The scholarly papers as reviewed and published by the Institute of Research Advances (IRA) are the views and opinions of their respective authors and are not the views or opinions of the IRA. The IRA disclaims of any harm or loss caused due to the published content to any party.

Institute of Research Advances is an institutional publisher member of Publishers International Linking Association Inc. (PILA-CrossRef), USA. The institute is an institutional signatory to the Budapest Open Access Initiative, Hungary advocating the open-access of scientific and scholarly knowledge. The Institute is a registered content provider under Open Access Initiative Protocol for Metadata Harvesting (OAI-PMH).

The journal is indexed \& included in WorldCat Discovery Service (USA), CrossRef Metadata Search (USA), WorldCat (USA), OCLC (USA), Open J-Gate (India), EZB (Germany) Scilit (Switzerland), Airiti (China), Bielefeld Academic Search Engine (BASE) of Bielefeld University, Germany, PKP Index of Simon Fraser University, Canada. 


\section{ABSTRACT}

This article reported a study of verb synonym's valency structure based on corpus data, which clearly shows the affiliation between the word item and its governing member. This study analyzed 'provide' and 'offer' in British National Corpus from the perspective of types and frequency distribution of valency structures, action elements, the relevance between valency structure and its meaning. The results indicate the valency structure is characteristic and closely interrelated with word's meaning. Therefore, this can give us some enlightenment in $h$ distinguishing verb synonyms effectively and promoting the English vocabulary teaching.

Keywords: Valency structure; Verb synonyms; Corpus; English vocabulary teaching

\section{Introduction}

Traditional linguistic research is limited by personal experience and intuition, and advocates that grammar and vocabulary belong to different systems. The emergence of corpus has injected a strong vitality into language research, making it a data-based positivist study. Through the analysis of a large number of real authentic linguistic data, corpus linguists have been paying close attention to the phrasal features in language, advocating that vocabulary and grammar are inseparable, and have obtained a series of research results, such as collocation (Sinclair 1991), extended unit of meaning (Sinclair 1996), pattern grammar (Hunton \& Francis 2000), lexical bundle (Biber, Conrad \& Cortes 2004), concgram (Cheng, Greaves \& Warren, 2006), description system of valency structure (Zhen Fengchao, Yang Feng 2015), etc. These studies are widely used in many fields, and provide new ideas for English language teaching.

Synonyms refer to words with the same or similar meaning in the same language. Teachers and learners often use dictionaries to distinguish them. However, the traditional dictionaries mainly provide the meaning of words, and have little effect on the specific use (Sinclair, 1991:7). In recent years, many researchers have tried to discriminate synonyms by means of corpus. However, they mostly distinguish them by word frequency, register, collocation, class connection and semantic prosody. The analysis is scattered and not systematic, which has little effect on discrimination and learning of synonyms in the process of teaching. What's more, most of the selected words are used in a single way, which can be effectively distinguished only by examining collocations. However, there are a large number of synonyms with complex structure and usage in the language, which need to be explained in detail.

The valency structure takes the word item as the core. It not only retains the words and parts of speech in the form grammar, but also increases the syntactic function of the valence grammar. The effective combination of words and structures can help learners understand the typical structure and usage of word items more clearly.

\section{Valency structure}

Valency grammar was proposed by French linguist Lucien Tesnière in the 1950s. Its main purpose is to show the syntactic and semantic relations between words. The valence of a verb is the number of action elements it dominates (Lu Jianming 1997:5). If we want to find out the valency structure of a 
verb, we just have to see the number of its action elements, and the common ones are zero valence verbs, monovalent verbs, bivalent verbs and trivalent verbs. Herbst et al. (2004: VII) compiled the first English valence dictionary, pointing out that words, like atoms, do not exist in isolation, but form larger units with other words. Teubert (2007:61) believes that valency grammar is a kind of continental grammar, which connects local grammar with common grammar.

Pattern grammar is a language description method proposed by Hunston \& Francis (2000) on the basis of COBUILD project. They regard vocabulary as the core of language and study language from a new perspective (Chen Gong, Liang Maocheng, 2017:18). Its main ideas include: vocabulary and grammar are inseparable; form and meaning are interrelated (Wang Yong, 2008:259). The specific operation is to summarize the types and words belonging to this type based on the observation of corpus data.

As two different language description systems, valency grammar and pattern grammar have obvious advantages and play a strong role in various fields of research, but they also have some limitations. On the one hand, pattern grammar is different from valency grammar in that it does not use syntactic labels, leaving only part of speech as a grammatical category. There may be ambiguity in this approach. On the other hand, previous studies on valency grammar are mostly based on introspective data and adopt a top-down research method, which is not consistent in terms of quantity, nature and classification of valency (Yuan Yulin, 2010). Therefore, some scholars suggest that the two should be combined, and corpus driven approach should be adopted to focus on word items, covering some specific words, parts of speech and syntactic functions.

\section{Research design}

The data analysis of this paper mainly takes provide and offer as an example to investigate the valency structure and differences of verb synonyms. In BNC, we search and analyze the valence structure of the provided verbs respectively, and explore their similarities and differences in order to improve the students' ability to use phrases properly, help them to apply them differently and provide enlightenment for the teaching of synonyms.

Because there are too many search lines in BNC (21978 of provide and 15408 of offer), only the first 600 items are selected for analysis, and the use of offer as a noun is excluded. In the analysis of the valency structure of provide and offer, the valency structure system in A Valency Dictionary of English (Herbst el al., 2004) and Collins COBUILD grammar patterns 1: verbs is used.

\section{Data Analysis}

4.1 Comparison of valency structure and frequency distribution

The verbs of provide and offer is searched in BNC, and get 21978 and 15408 index lines respectively, and extract 600 of them. After screening, 422 and 297 effective index lines with the two as the main predicate verbs are obtained. The specific results of the valence structure are as follows. 
IRA-International Joumal of Education $\Xi^{0}$ Multidisciplinary Studies

Table 1 The Types and Frequency Distribution of Valency Structure of Provide

\begin{tabular}{|c|c|c|c|}
\hline Valency structure & Frequency & Percentage(\%) & Example \\
\hline Bivalent structure & \multicolumn{3}{|c|}{} \\
\hline Sub V obj & 220 & 52.13 & Mr Williams should provide a real altemative to Belville. \\
\hline Sub V for obj & 8 & 1.9 & RemenberI depend upon you fitly to provide for her \\
\hline Sub V against obj & 2 & 0.4 & banks will provide against masses of doubtful debts \\
\hline Sub V obj-that & 2 & 0.4 & $\begin{array}{c}\text { section 322A of the companies act provides that such a } \\
\text { transation is voidable. }\end{array}$ \\
\hline Trivalent structure & 111 & 26.3 & $\begin{array}{c}\text { local government provide an exciting experience for young } \\
\text { people. }\end{array}$ \\
\hline Sub V obj for nom & 32 & 7.58 & $\begin{array}{c}\text { her instinctive feeling for him makes provide him with some } \\
\text { hope of return. }\end{array}$ \\
\hline Sub V obj with nom & 31 & 7.34 & $\begin{array}{c}\text { They quickly provide the necessary interface to network } \\
\text { PCs, workstations. }\end{array}$ \\
\hline Sub V obj to nom & 15 & 3.55 & $\begin{array}{c}\text { Treatment centers provide services to meet the particular } \\
\text { needs. }\end{array}$ \\
\hline Sub V obj to-vb-inf & 1 & 0.24 & The hall provide the commual eating and drinking space \\
\hline Sub V obj nom & 1 & &
\end{tabular}

From the table 1, we can see that there are nine kinds of valency structure in the active sentence of provide, including four kinds of bivalent structure and five kinds of trivalent structure. The most commonly used structure was bivalent structure sub-v-obj, with a frequency of 220 (52.13\%), followed by trivalent structure sub-v- obj for NOM, with 111 occurrences $(26.30 \%)$. It should be noted that the frequency of sub-v- obj to NOM and sub- V -obj with NOM are 31 and 32 times (7.58\% and 7.34\%) respectively. From this we can know that although the typical grammatical form of provide is $\mathrm{V}$ - $\mathrm{n}$ with $\mathrm{n}$, there are also a large number forms of $\mathrm{V} n$ to $\mathrm{n}$. While in traditional vocabulary teaching, teachers make qualitative analysis based on introspective data and experience, and tell students that provide only can be applied in the structure provide somebody with something and provide something for body. This is contrary to the real corpus data, which further confirms the scientificity and necessity of corpus research.

Table 2 The Types and Frequency Distribution of Valency Structure of Offer

\begin{tabular}{|c|c|c|c|}
\hline Valency structure & frequency & percentage $(\%)$ & Example \\
\hline \multicolumn{4}{|l|}{ Bivalent structure } \\
\hline Sub V Obj & 143 & 48.15 & Most now offer free counselling. \\
\hline Sub V to-vb-inf & 22 & 7.41 & By day we'll offer to show you around the island. \\
\hline Sub V up Obj & 1 & 0.34 & We offer up this Holy Rosary for the repose of the soul of James. \\
\hline \multicolumn{4}{|l|}{ Trivalent structure } \\
\hline Sub V Obj to nom & 55 & 17.51 & He doesn't offer anything to the game. \\
\hline Sub V Obj nom & 46 & 15.49 & We'll be more than happy to offer you our FREE service. \\
\hline Sub V Obj for nom & 26 & 8.75 & But no-one can offer any hope of a proper home for Heidi. \\
\hline Sub V Obj to-vb-inf & 7 & 2.36 & Workers offer co-therapists to work with a family. \\
\hline
\end{tabular}

The valency structure of offer in the active sentence includes two kinds of structures: bivalent and trivalent. Consistent with provide, the most commonly used structure is sub-v -obj, which occurs 143 times $(48.15 \%)$. The second is the trivalent structure sub- V -obj to NOM and sub -V -obj NOM, with frequency of $52(17.51 \%)$ and $46(15.49 \%)$ respectively. The frequencies of bivalent sub-v -to VB inf 
IRA-International Joumal of Education $\Xi^{\circ}$ Multidisciplinary Studies

and trivalent sub-v obj for NOM were close, accounting for $7.41 \%$ and $8.75 \%$ of the total frequency, respectively. The most common valency structures include bivalent sub-v up obj (once) and trivalent sub-v -obj to VB- inf (7 times).

Table 3 The Comparision of the Valency Structure Between Provide and Offer

\begin{tabular}{|c|c|c|}
\hline \multicolumn{1}{|c|}{ Valency structure } & provide & offer \\
\hline Bivalent structure & $52.13 \%$ & $48.15 \%$ \\
\hline Sub V Obj & $1.90 \%$ & \\
\hline Sub V for Obj & $0.4 \%$ & \\
\hline Sub V against Obj & $0.4 \%$ & $7.41 \%$ \\
\hline Sub V Obj-that & & $0.34 \%$ \\
\hline Sub V to-vb-inf & \\
\hline Sub V up Obj & $26.30 \%$ & $8.75 \%$ \\
\hline Trivalent structure & $7.58 \%$ & $17.51 \%$ \\
\hline Sub V Obj for nom & $7.34 \%$ & $2.36 \%$ \\
\hline Sub V Obj with nom & $3.55 \%$ & $15.49 \%$ \\
\hline Sub V Obj to nom & $0.24 \%$ & \\
\hline Sub V Obj to-vb-inf & Sub V Obj nom &
\end{tabular}

Through the analysis, the author has the following findings: first, there are different types of valency structure. Both of them are used in bivalent and trivalent structure, but provide is the most abundant (9 kinds), followed by offer (7 kinds), and sub- V for obj, sub -V against obj and sub-V obj that are exclusive to provide; sub $-\mathrm{V}$ to $\mathrm{VB}$ inf and sub $-\mathrm{V}$ up obj are exclusive to offer. Secondly, the frequency distribution of trivalent structure is quite different, which indicates that they are different in usage tendency.

\subsection{Relevance between valency structure and meaning}

There are four kinds of exclusive structures: (1). Sub $-\mathrm{V}$ for obj, the subject is usually a person or organization with the ability to think, at this time, provide means to prepare for...; (2). The verb in sub -V against obj expresses the meaning of "prevention"; (3). Sub-v obj in this structure, the subject is usually a legal regulation. The main meaning of the regulation is explained by the clause after that, and the verb "provide" means "provision"; (4). The verb in sub -V obj with NOM means "provide". There are two kinds of exclusive valency structure of offer: (1) In the sub -V to VB inf valency structure, the subject refers to the person, which means "willing to do sth". For example, the gardener comes three times a week and did not offer to mend it. (2) Sub- V up obj appears only once. By consulting $A$ Valency Dictionary of English (Herbst et al., 2004), it is found that Herbst and others have classified it as idiomatic phrasal verbs, and its meaning can be summarized as "expression, presentation and contribution". 
IRA-International Joumal of Education $\Xi^{\circ}$ Multidisciplinary Studies

Table 4 The meaning of Provide and Offer in its Exclusive Valency Structure

\begin{tabular}{|c|c|c|}
\hline Word item & Valency structure & Meaning \\
\hline \multirow{3}{*}{ provide } & Sub V for Obj & prepare for \\
\cline { 2 - 3 } & Sub V against Obj & prevention \\
\cline { 2 - 3 } & Sub V Obj-that & provision \\
\cline { 2 - 3 } & Sub V Obj with nom & offer \\
\hline \multirow{3}{*}{ offer } & Sub V to-vb-inf & be willing to do something \\
\cline { 2 - 3 } & Sub V up Obj & presentation/contribution \\
\hline
\end{tabular}

\subsection{Enlightenment for teaching synonyms}

In traditional vocabulary teaching, teachers mainly rely on dictionary interpretation, and explain certain fixed usage by experience, which is separated from specific context. However, the meaning of the synonyms is similar, which has caused great trouble to students. For example, many students only know that the verbs of the type of offer have the meaning of providing, which can not be distinguished in the specific application. The analysis shows that although they are described as synonyms, their valence structures are different. These pricing structures show different usage and distinctive characteristics. By learning the valency structure, learners can grasp vocabulary comprehensively and accurately and understand their differences, and choose corresponding words according to the context.

First, there are obvious differences in the types and frequencies of the two valency structures, among which provide pricing structure is the most abundant, and the usage is the most diverse, and the structure of offer is the second. The analysis of the proportion of different structures can help learners understand the idiomatic expression of different verbs. Except for sub- V obj, the two expressions are in trivalent structure, namely sub -V obj for NOM, sub -V- obj to NOM and sub -V- obj NOM; sub -V-obj with NOM.

Secondly, the valency structure and corresponding meaning are related. The study finds that the meaning of providing verbs is not only limited to "provision", and different valency structures are related to different meanings. Matching the valency structure with meaning can help learners master typical usage and improve the ability of using phrases. On the one hand, the valency structure itself is closely related to the meaning, and there is often a one-to-one linear relationship. For example, sub provide against obj means "prevention". Understanding these valency structures can help us quickly master the usage of the word. On the other hand, the meaning of the valency structure also changes with the different o action element, namely collocation. The same valency structure may correspond to different meanings; collocation is the main distinguishing factor.

\section{Conclusions}

The corpus contains quantitative real linguistic data which can provide students with authentic expression and real use. This paper studies the valency structure of verbs based on corpus, extracts real data, investigates the valence structure of verb provide and offer, and makes a concrete analysis from the structure types, quantity, valency structure and relevance of meaning, which enables us to have a comprehensive understanding of both. The analysis shows that the valency structure of verbs has different characteristics, which can provide strong support for the discrimination of synonyms, and help 
learners to distinguish and use them properly.

First of all, they are different in terms of the types and frequency of the valency structure, Secondly, the valency structure and its meaning are closely related, and the combination of valency structure and meaning can help learners to quickly grasp the usage of vocabulary. At the same time, the semantic tendency of collocation is one of the factors we can use to distinguish the synonyms in the valency structure. All of these indicate that learning valency structure can help learners master typical usage and effectively distinguish synonyms. The author suggests that in the teaching of synonyms, corpus data containing real context should be introduced, and the focus of learning should be on the valency structure, so as to help learners master the correct and authentic language expression.

\section{References}

[1]. Biber, D., S. Conrad \& V. Cortes. 2004. If you look at ... : Lexical bundles in university teaching and textbooks [J]. Applied Linguistics ( 3 ) : 71-405.

[2]. Cheng, W., C. Greaves \& M. Warren. 2006. From n-gram to skipgram to concgram [J]. International Journal of Corpus Linguistics ( 4 ) : 411-433.

[3]. Davidse, Kristin. (1998). Gill Francis, Susan Hunston and Elizabeth Manning (eds.) Collins cobuild grammar patterns 1: verbs. Functions of Language, 5(1), 97-99.

[4]. Hunston, S. \& G. Francis. 2000. Pattern Grammar: A Corpus -Driven Approach to the Lexical Grammar of English [M].Amsterdam: John Benjamins

[5]. Herbst, T., D. Heath, F. Roe \& D. Gotz. 2004. A Valency Dictionary of English: A Corpus-Based Analysis of the Complementation Patterns of English Verbs, Nouns and Adjectives [M]. Berlin/New York: Mouton de Gruyter.

[6]. Sinclair, J. 1991. Corpus, Concordance, Collocation [M]. Ox ford: Oxford University Press.

[7]. Sinclair, J. 1996. The search for units of meaning [J]. Textus ( 4 ) : 75-106

[8]. Teubert, W. 2007. Sinclair, pattern grammar and the question of hatred[A]. International journal of corpus linguistics, 12(2), 223-248.

[9]. Chen gong., \& Liang Maocheng. (2017)..The Origin, Features and Applications of Pattern Grammar[J]. Foreign Language Research, (01):17-24. (In Chinese)

[10].Liu Guobing.,\& Du Yaping. (2017). A corpus- driven study of verb valency pattern based on APPOINT [J].Journal of Tianjin Foreign Studies University, 24(06):1-7+78.(In Chinese)

[11].Wang Yong.(2008). On the borderline between grammar and lexis:A review of pattern grammar[J].Contemporary linguistics, (03):257-266+286

[12].Wang Lin. (2019). A study of synonyms based on COCA and BNC-----Taking endure and bear as an example. Think Tank Era(51),217-218(In Chinese)

[13].Zheng Fengchao., \& Wang Hua. (2010). The application of CLEC in foreign language teaching: Thoughts and Methods [J]. Foreign Language World, 72-77+90. (In Chinese)

[14].Zheng Fengchao., \& Xu Fanghong. (2017). Collovalency: Investigating the interaction of "consider" and its Valency Patterns in learner English [J]. Foreign Language and Translation, 24(04):44-50+98. (In Chinese)

[15].Zheng Fengchao.(2017). Exploring the Integration of Valency Pattern and Meaning Corpus-driven Approach [J]. Foreign Language Research, 13-18. (In Chinese)

[16].Zheng Fengchao., \& Yang Feng. (2016). The application of valency pattern and collocation valency in English vocabulary teaching[J]. Foreign Language World.(04):35-42. (In Chinese) 